\title{
ХРОНИЧЕСКИЕ ЗАБОЛЕВАНИЯ ПЕЧЕНИ В ПЕРИОД ПАНДЕМИИ COVID - 19
}

\author{
А.Р. Рейзис, Ж.Б. Понежева, В.В. Макашова, Х.Г. Омарова, \\ И.В. Маннанова, А.В. Горелов \\ ФБУН «Центральный НИИ Эпидемиологии» Роспотребнадзора РФ
}

PЕЗЮМЕ. В обзоре обобщены последние данные о состоянии проблемы хронических заболеваний печени (Х3П) на фоне пандемии COVID - 19.Показано, что ХЗП, не предрасполагая к инфицированию, отягощают исходы и прогноз SARS-CoV-2 и связаны с её более тяжелым течением и большей летальностью. При циррозе печени количество баллов по шкале Чайлд-Пью, рост билирубина и уровень изменения АЛТ/АСТ имеют предсказательную силу в отношении летальности, приводя к декомпенсации. Острейшая проблема трансплантации печени в период пандемии связана не только с медицинскими, но социальными, финансовыми и другими условиями конкретных стран. В настоящее время существует лишь несколько временных национальных практических рекомендаций в отдельных странах, которые не могут рассматриваться как единое руководство.

Ключевые слова: хронические заболевания печени (X3П), пандемия COVID - 19, течение новой коронавирусной инфекции, взаимовлияние X3П и SARS-CoV-2.

Пандемия нового коронавируса COVID - 19 переключила медицину всех стран мира на первоочередную борьбу с этой инфекцией. Однако атака COVID - 19 не отменила болезней, которыми страдало человечество до развития пандемии. И, по мере того, как благодаря совместным усилиям медицинского сообщества, пандемия стабилизируется, а в ряде стран начинает идти на убыль, назревает необходимость вернуть внимание к пациентам с другими заболеваниями и оценить их взаимодействие с новой беспрецедентной инфекцией, об особенностях которой как per se, так и в сочетании с другими патологическими состояниями, нам известно ещё очень мало.

В ходе пандемии стало очевидным, что COVID - 19 это не просто острая респираторная инфекция, но заболевание с полиорганным системным поражением, в том числе такого важнейшего органа как печень. Этот вопрос был освещён нами в специальных обзорах [1,2], основанных на обобщении имевшихся на тот момент данных литературы. Было показано, что печень наряду с респираторным трактом активно участвует в патологическом процессе, а течение и исход COVID-19 тесно связаны с частотой и степенью поражения печени этой инфекцией. Однако, проблемы пациентов с 
хроническими заболеваниями печени (ХЗП) на фоне пандемии COVID-19 остаются нерешёнными и требуют изучения.

Данное сообщение посвящено обобщению и осмыслению имеющихся сведений о течении и взаимовлиянии ХЗП и COVID-19 в качестве состоявшейся суперинфекции и/или высокой угрозы её наслоения.

В специальном обзоре, посвящённом этой проблеме, Covalic A J et al. [3] отмечают, что истинная распространенность и клиническая значимость основных ХЗП при COVID19 остаются недостаточно определенными. В этот систематический обзор и метаанализ они включили 74 клинических исследования, которые были выявлены после тщательного поиска литературы в трех базах данных. Распространенность пациентов с ХЗП (73 исследования, 24299 пациентов) составила 3\% среди всех пациентов с COVID-19. Удельный вес пациентов с ХЗП был аналогичен как в COVID-19 положительной популяции, так и в отрицательной (объединенное OR 0,79 [95\% CI 0,60, 1,05], p = 0,10). Эти данные могут свидетельствовать о том, что наличие ХЗП не обязательно предполагает заражение новой вирусной инфекцией SARS-CoV-2. Однако показано, что присутствие ХЗП было в значительной степени связано с более тяжелым течением COVID-19 (объединенное ИЛИ 1,48 [95\% ДИ 1,17, 1,87], $\mathrm{p}=0,001$ ) и более высокой общей смертностью (объединенное ИЛИ 1,78 [95\% ДИ 1,09, 2,93], p = 0,02). Кроме того, отмечена тенденция к увеличению числа обращений в ОРИТ и необходимости применения инвазивной искусственной вентиляции легких у пациентов с новой коронавирусной инфекцией на фоне ХЗП. Авторы отмечают, что на сегодняшний день клиническое значение хронических заболеваний печени среди пациентов с инфекцией COVID-19 остается достаточно неопределенным. Однако неоспоримо, что наличие ХЗП отягощает исходы и прогноз SARS-CoV-2, так как чётко связано с более тяжелым течением инфекции COVID-19 и большей летальностью.

B обзоре Portincasa P et al. [4] отмечается, что механизмы повреждения печени при новой коронавирусной инфекции являются сложными и включают в себя как прямое повреждение холангиоцитов, так и другие сопутствующие состояния (использование противовирусных препаратов, системный воспалительный ответ, респираторный дистресс-синдром, гипоксия, сепсис, полиорганная дисфункция и др.). У больных при заболевании COVID-19 может наблюдаться повреждение печени. Однако авторы особо подчёркивают, что требуется дополнительное внимание, если пациенты уже имеют в анамнезе ХЗП, что ухудшает прогноз, и удлиняет время пребывания в больнице.

Масштабное целенаправленное изучение вопроса о зависимости COVID-19 от сопутствующих заболеваний, в частности, у пациентов с ранее существовавшим 
хроническим заболеванием печени (ХЗП) предприняла большая группа исследователей Sarin S K et al. [5]. Ученые отмечают важность проведённой работы в связи с тем, что вопрос в значительной степени неизвестен. Они изучили показатели поражения печени на фоне SARS-Cov-2 у пациентов с ХЗП, с циррозом или без него. Были собраны данные из 13 стран Азии о пациентах с ХЗП с подтвержденным диагнозом: COVID-19.

Всего было зарегистрировано 228 пациентов (185 ХЗП без цирроза и 43 с циррозом). Наиболее распространенными были жировая болезнь печени (113 чел; 61\%) и вирусная этиология поражения печени (26 чел; 60\%). При ХЗП без цирроза в сравнении с пациентами в цирротической стадии сахарный диабет встречался с частотой 57,7\% против $39,7 \%$ - без него (OR=2,1 (1,1-3,7), p=0,01), а ожирение - 64,3\% против $17,2 \%,(\mathrm{OR}=8,1$ $(1,9-38,8), \mathrm{p}=0,002)$ соответственно. У 43\% пациентов с ХЗП без цирроза печени изменения со стороны печени на фоне COVID -19 были расценены как острое повреждение печени без явлений её недостаточности. У 20\% больных с циррозом печени развились: нарастание хронической печеночной недостаточности у 5 чел $(11,6 \%)$ или острая декомпенсация у 4 (9\%)). Связанные с поражением печени осложнения увеличивались $(\mathrm{p}<0,05)$ со стадией заболевания печени следующим образом: балл по шкале Чайлд- Пью, равный 9 или более, предсказывал высокую смертность (AUROC 0,94, ЧСС = 19,2 (95 ДИ 2,3-163,3), p<0,001, чувствительность 85,7\% и специфичность 94,4\%). При декомпенсированном циррозе поражение печени было прогрессирующим у 57\% пациентов с 43\% летальностью. Рост билирубина и уровень изменения АСТ / АЛТ имел предсказательную силу в отношении летальности среди пациентов с циррозом. Авторы приходят к однозначному выводу: инфекция SARS-Cov-2 вызывает значительное дополнительное повреждение печени у пациентов с ХЗП, приводя к декомпенсации у 1/5 части пациентов с циррозом печени и существенному ухудшению клинического статуса уже декомпенсированного цирроза.

Интересные данные в контексте общей гастроэнтерологии приводят в своей работе «COVID-19 - больше, чем респираторное заболевание: взгляд гастроэнтеролога» Konturek PC, Harsch IA, Neurath MF, Zopf Y [6]. Авторы расценивают пандемию коронавируса-2 (SARS-CoV2) как наиболее драматичное событие со времен Второй мировой войны. При этом с их точки зрения, гастроэнтерология является одной из медицинских дисциплин, которая наиболее связана с этой вирусной инфекцией. Известно, что COVID-19 вызывает у некоторых пациентов типичные симптомы энтерита, такие как диарея или боль в животе. Есть также доказательства того, что эта инфекция может привести к повреждению печени и поджелудочной железы. Поскольку вирус SARS-CoV-2 был обнаружен в кале, не исключается возможность фекально-орального пути передачи. Более того, показано, что 
ангиотензин-превращающий фермент 2 (АПФ-2) вирусного рецептора (ACE2) высоко экспрессируется в желудочно-кишечном тракте и обеспечивает проникновение вируса в желудочно-кишечный эпителий, как продемонстрировано in vitro и in vivo. В связи с этим, по мнению авторов, пандемия COVID-19 не может не оказывать влияния на проблемы гастроэнтерологии в более широком смысле: повседневную врачебную практику и рабочие процессы в эндоскопии, что приводит к резкому сокращению процедур скрининга и наблюдения. COVID-19 влияет на терапию пациентов с воспалительными заболеваниями кишечника (ВЗК), особенно тех, кто использует высокие дозы кортикостероидов, иммунодепрессантов и биопрепаратов, а пациенты с ранее существовавшим заболеванием печени, особенно с неалкогольной жировой болезнью печени (НАЖБП) с фиброзом или циррозом печени, подвержены высокому риску тяжелой формы COVID-19.

Особенно неблагоприятна выраженная при COVID-19 склонность к тромбоцитопении, исходно характерная для пациентов с циррозом печени. Случай подобного сочетания приводят в своей работе Artru F, Alberio L, Moradpour D, Stalder G. [7]. Авторы сообщают о пациенте с коронавирусной болезнью (COVID-19) и декомпенсированным циррозом печени, у которого получен благоприятный исход тяжелой иммунной тромбоцитопенической пурпуры (ИТП) после введения внутривенного иммуноглобулина и высоких доз дексаметазона. Настоящий случай, по мнению авторов, должен насторожить врачей и заставить предполагать возможность ИТП в случае глубокой тромбоцитопении у пациентов с COVID-19 на фоне цирроза печени.

Отдельной стороной повреждения печени на фоне COVID-19 у пациентов с предшествующим ХЗП является лекарственно - индуцированное поражение (ЛИПП). В работе Xie YB, Wang SY, Zhang C, Wang FS [8] отмечается, что поскольку в настоящее время нет конкретных лекарств для лечения COVID-19, множество потенциально эффективных противовирусных препаратов, вакцин, клеточной терапии, традиционной китайской медицины и других методов находятся на стадии клинических испытаний. Они, в свою очередь, могут вести к повреждению печени, в связи с чем последнее является весьма многофакторным и частым осложнением у пациентов, в особенности среди пациентов, уже имеющих ХЗП, что может дополнительно повлиять на исход заболевания. Авторы отмечают, что патогенез COVID-19 в сочетании с повреждением печени в существующих исследованиях до сих пор неясен, а соответствующие рекомендации и экспертные заключения недостаточны для клинической диагностики и лечения. В настоящее время речь идёт только о пристальном внимании к состоянию печени у этих 
больных и доступной симптоматической терапии. Изучение этих вопросов, по мнению авторов, является актуальной задачей для дальнейших исследований.

Особняком среди больных с ХЗП и разразившейся пандемией новой короновирусной инфекции стоит проблема пациентов, связанных с трансплантацией печени (ТП): как тех, что уже перенесли ТП и находятся на соответствующей иммуносупрессивной терапии, так и ожидающих трансплантацию. Проблема приковала к себе внимание и стоит столь остро не потому, что это частая ситуация, но в связи с абсолютной неотложностью её решения и для конкретного пациента, и для всего многочисленного звена причастных к этому вопросу учреждений (медицинских, социальных и прочих). Этим объясняется тот факт, что в потоке медицинской литературы, посвящённой COVID-19, проблема ТП активно обсуждается.

В работе Dahlqvist G, Ciccarelli O, Van Vlierberghe H, et al. [9] в контексте трансплантации печени (ТП) в эпоху пандемии выражается обеспокоенность по поводу нехватки и безопасности донорства органов, этики использования ограниченных ресурсов здравоохранения для ТП, включая пожертвования, критериев отбора пациентов для ТП в период эпидемии и риска заражения de novo COVID-19 в процессе ожидания, во время и после ТП. В сообщении дается несколько рекомендаций, чтобы попытаться смягчить вредное воздействие, которое эта пандемия уже оказывает и ещё может оказать на всю совокупность проблем, связанных с ТП на фоне пандемии COVID -19. При этом авторы подчёркивают, что рекомендации носят не только временный, но и сугубо локальный характер, касающийся конкретных условий конкретной страны (в данном случае Бельгии).

El Kassas M, Alboraie M, Al Balakosy A, et al. [10] делают попытку обобщить рассматриваемую проблему. Авторы отмечают, что трансплантация печени считается окончательным решением для пациентов с терминальной стадией хронического заболевания печени или острой печеночной недостаточностью. Пациенты с трансплантацией печени и кандидаты на неё нуждаются в особом уходе, начиная с предоперационной подготовки и заканчивая хирургическим вмешательством и уходом после операции. Трансплантированные пациенты должны получать иммуносупрессивную терапию для предотвращения отторжения. Такое состояние подавления иммунитета может предрасполагать к различным типам инфекций у реципиентов печени. В условиях, когда мир страдает от пандемии, вызванной новым штаммом семейства коронавирусов SARS-CoV-2, необходимы определенные меры инфекционного контроля для защиты от заражения COVID-19 пациентов с ослабленным иммунитетом и уязвимых пациентов, включая кандидатов на трансплантацию печени и реципиентов. Ограничение не трансплантационных плановых хирургических процедур, ведение пациентов с 
трансплантацией в отдельных поликлиниках и стационарных отделениях может предотвратить передачу инфекции как пациентам, так и работникам здравоохранения. Телемедицина может помочь в сортировке пациентов для выявления симптомов COVID19. Управление иммуносупрессивной терапией и лекарственным взаимодействием у реципиентов печени, инфицированных COVID-19, должно осуществляться с огромной осторожностью, чтобы предотвратить отторжение трансплантата и эффективно лечить вирусную инфекцию.

Но это общие соображения, которые следует учитывать. В основном же в настоящее время существует лишь несколько отдельных временных практических рекомендаций в таких странах, как Бельгия, Индия, Турция, Китай и даже для группы азиатскотихоокеанских стран AASLD, но все они не могут рассматриваться как единое руководство, так как исходят из местных медицинских и социальных условий. Такие авторы, как Akdur A, Karakaya E, Ayvazoglu Soy EH, et al. [11] или Kabaçam G, Dayangaç M, Üçbilek E, et al. [12] прямо утверждают это, стремясь представить лишь набор фактов и материалов для обдумывания и возможного согласования.

Taк, Akdur A et al. [11] в работе из Китая представили 583 пациента, перенесших трансплантацию какого-либо органа, которые были госпитализированы в клиники и отделения неотложной помощи в период с 1 марта по 1 мая 2020 года. Семьдесят четыре из них были реципиентами трансплантата печени (46 мужчин, 28 женщин, из которых было 14 детей и 60 взрослых больных). Авторы констатируют, что пациенты, перенесшие трансплантацию, также пострадали во время пандемии COVID-19. Однако смертельных случаев не наблюдалось. В своих рекомендациях авторы считают важным, чтобы реципиенты трансплантата были предупреждены о соблюдении личной гигиены и тщательно контролировались центрами трансплантации органов. Если есть показания к госпитализации, их следует проводить в изолированные отделения, без каких-либо агрессивных изменений в иммуносупрессивных дозах без абсолютной необходимости.

Sahin TT et al. [13] также подчёркивают, что пациенты с заболеваниями печени нуждаются в особом внимании и постоянном наблюдении, а кандидаты на пересадку печени или уже получившие ТП нуждаются в особом уходе.

Очевидно, что острейшая проблема тактики ведения пациентов, перенесших ТП или находящихся на стадии её ожидания в условиях пандемии COVID-19, не имеет сегодня общего решения. Могут обсуждаться вопросы отношения к обязательной для этих пациентов иммуносупрессивной терапии, допустимости и степени её коррекции в определённых обстоятельствах и т.д. Все остальные стороны проблемы жёстко завязаны 
на конкретные национальные условия и возможности и имеют только временное и местное решение.

ВЫВОДЫ:

1. Наличие ХЗП неблагоприятно для суперинфекции COVID-19, отягощает исходы и прогноз заболевания, так как связано с более тяжелым его течением и большей летальностью.

2. Механизмы повреждения печени при COVID -19 являются сложными и включают в себя как прямое повреждение холангиоцитов, так и системный воспалительный ответ, респираторный дистресс-синдром, гипоксию, сепсис, полиорганную дисфункцию и др. Наличие предшествующего ХЗП является дополнительным предрасполагающим фактором для реализации тяжелого течения.

3. Степень поражения печени достоверно увеличивается $(\mathrm{p}<0,05)$ со стадией заболевания печени: так, балл по шкале Чайлд-Пью, равный 9 или более, рост билирубина и уровень изменения АСТ/АЛТ имеют предсказательную силу в отношении летальности среди пациентов с циррозом печени, приводя к декомпенсации и/или существенному ухудшению клинического статуса уже декомпенсированного цирроза.

4. Существуюие рекомендации и экспертные заключения недостаточны для клинической диагностики и лечения поражений печени при сочетании XЗП и COVID - 19. Необходимо пристальное внимание к состоянию печени у этих больных и использование доступной симптоматической терапии. Изучение этих вопросов - актуальная задача для дальнейших исследований.

5. Многостороннее вредное воздействие, которое COVID-19 уже оказывает и ещё окажет на всю совокупность проблем, связанных с ТП на фоне пандемии, обусловлена не только медицинскими, но социальными, финансовыми и другими условиями конкретных стран. В настоящее время существует лишь несколько временных практических рекомендаций в отдельных странах, но все они не могут рассматриваться как единое руководство.

\section{ЛИТЕРАТУРА}

1. Рейзис А. Р., Понежева Ж. Б., Макашова В. В., Омарова Х. Г., Маннанова И. В., Горелов А. В. 2020. ПОРАЖЕНИЕ ПЕЧЕНИ ПРИ COVID-19. COVID19PREPRINTS.MICROBE.RU. https://doi.org/10.21055/preprints-3111760

2. Маннанова И. В., Понежева Ж. Б., Макашова В. В., Омарова Х. Г., Рейзис А. Р., Горелов А. В. 2020. НЕКОТОРЫЕ АСПЕКТЫ ПОВРЕЖДЕНИЯ ПЕЧЕНИ ПРИ ИНФЕКЦИИ, ВЫЗВАННОЙ SARS-CoV-2. COVID19-PREPRINTS.MICROBE.RU. https://doi.org/10.21055/preprints-3111776 
3. Kovalic AJ, Satapathy SK, Thuluvath PJ. Prevalence of chronic liver disease in patients with COVID-19 and their clinical outcomes: a systematic review and meta-analysis [published online ahead of print, 2020 Jul 28]. Hepatol Int. 2020;10.1007/s12072-020-10078-2. doi:10.1007/s12072-020-10078-2

4. Portincasa P, Krawczyk M, Machill A, Lammert F, Di Ciaula A. Hepatic consequences of COVID-19 infection. Lapping or biting?. Eur J Intern Med. 2020;77:18-24. doi:10.1016/j.ejim.2020.05.035

5. Sarin SK, Choudhury A, Lau GK, et al. Pre-existing liver disease is associated with poor outcome in patients with SARS CoV2 infection; The APCOLIS Study (APASL COVID-19 Liver Injury Spectrum Study) [published online ahead of print, 2020 Jul 4]. Hepatol Int. 2020;111. doi:10.1007/s12072-020-10072-8

6. Konturek PC, Harsch IA, Neurath MF, Zopf Y. COVID-19 - more than respiratory disease: a gastroenterologist's perspective. $\mathrm{J}$ Physiol Pharmacol. 2020;71(2):10.26402/jpp.2020.2.02. doi:10.26402/jpp.2020.2.02

7. Artru F, Alberio L, Moradpour D, Stalder G. Acute immune thrombocytopaenic purpura in a patient with COVID-19 and decompensated cirrhosis. BMJ Case Rep. 2020;13(7):e236815. Published 2020 Jul 7. doi:10.1136/bcr-2020-236815

8. Xie YB, Wang SY, Zhang C, Wang FS. Zhonghua Gan Zang Bing Za Zhi. 2020;28(6):523-527. doi:10.3760/cma.j.cn501113-20200416-00190. [Прогресс исследования клинических особенностей и механизмов применения COVID-19 в сочетании с повреждением печени]

9. Dahlqvist G, Ciccarelli O, Van Vlierberghe $\mathrm{H}$, et al. Liver transplantation during the COVID-19 epidemic : recommendations from the Belgian Liver Intestine Transplant Committee (BeLIAC). Acta Gastroenterol Belg. 2020;83(2):340-343

10. El Kassas M, Alboraie M, Al Balakosy A, et al. Liver transplantation in the era of COVID-19. Arab J Gastroenterol. 2020;21(2):69-75. doi:10.1016/j.ajg.2020.04.019

11. Akdur A, Karakaya E, Ayvazoglu Soy EH, et al. Coronavirus Disease (COVID-19) in Kidney and Liver Transplant Patients: A Single-Center Experience. Exp Clin Transplant. 2020;18(3):270-274. doi:10.6002/ect.2020.0193

12. Kabaçam G, Dayangaç M, Üçbilek E, et al. The COVID-19 pandemic: Clinical practice advice for gastroenterologists, hepatologists, and liver transplant specialists. Turk J Gastroenterol. 2020;31(5):348-355. doi:10.5152/tjg.2020.2041

13. Sahin TT, Akbulut S, Yilmaz S. COVID-19 pandemic: Its impact on liver disease and liver transplantation. World J Gastroenterol. 2020;26(22):2987-2999. doi:10.3748/wjg.v26.i22.2987 